Classification

Physics Abstracts

$05.40-05.50$

\title{
Current-voltage characteristic of a non linear resistor network
}

\author{
A. Gilabert, S. Roux ${ }^{+}$and E. Guyon ${ }^{+}$ \\ Laboratoire de Physique de la Matière Condensée, UA 190, Université de Nice, 06034 Nice Cedex, France \\ + ESPCI UA 857, 10 rue Vauquelin, Paris Cedex 05, France
}

(Reçu le 19 juin 1987, accepté le 27 juillet 1987)

\begin{abstract}
Résumé.- Nous présentons des mesures courant-tension $I(V)$ d'un réseau dont les liens sont constitués de dipoles ayant des caractéristiques locales à seuil (courant linéaire en tension au-delà du seuil $v_{\mathrm{g}}$ et nul en dessous). En présence de désordre dans la distribution des seuils $v_{g}$, le courant total $I$ est quadratique en fonction de la tension $V$ aux bornes du réseau confirmant des prévisions théoriques de Roux et Herrmann.
\end{abstract}

\begin{abstract}
Experimental I $V$ ) characteristics of a lattice are reported. Each bond of the lattice is a non linear resistor characterized by a threshold $v_{\mathrm{g}}$ for the potential drop below which it becomes an insulator. If the thresholds are randomly distributed, the current $I$ flowing through the lattice is a quadratic function of the voltage $V$, as predicted by Roux and Herrmann.
\end{abstract}

Recent studies of non linear two dimensional electrical networks have extended the range of application of problems of percolation type $[1,2]$. The interest is motivated in part by the analogy [3] between such electrical networks and mechanical or hydrodynamical two dimensional random media $[4,5]$. Recently, Roux and Herrmann [6] have calculated the currentvoltage characteristic $I(V)$ of an electrical network where each bond is a non linear resistor characterized by a threshold value $v_{\mathrm{g}}$ for the the potential drop below which it becomes an insulator. If the thresholds $v_{\mathrm{g}}$ are randomly distributed, the current $I$ flowing through the lattice of such bonds is a non linear function of the voltage $V$. The quadratic dependence observed then comes from the increasing number of passing bonds with applied voltage.

In this note, the numerical results of reference [6] are illustrated by experimental measurements of the $I(V)$ characteristic of a non linear resistor network. Two dimensional square lattices tilted by $45^{\circ}$ of $m \times n$ Zener diodes with $m, n \leq 29$ have been realized (Fig.1). The diodes are chosen among 7 different populations of 100 diodes and randomly distributed as the bonds of the network. A resistor $(r=1000 \Omega)$ is mounted in series with each diode. Figure 2 shows the $I(V)$ characteristic of each of the seven kinds of diodes in series with $r$. The current is very small below the threshold $v_{\mathrm{g}}$ and above $v_{\mathrm{g}}$ it increases with an asymptotic resistance of $1000 \Omega$. The threshold values $v_{g}$ spread between $1 \mathrm{~V}$ and $3 \mathrm{~V}$. The voltage across the network is measured as a function of the total bias current $I$.

The $I(V)$ characteristic of a $10 \times 24$ network is shown in the insert of figure 3. Below $V_{\mathrm{g}}=20 \mathrm{~V}$, there is almost no current in the network. In an intermediate range of voltages, the curve is parabolic. It becomes linear at high voltage. This behavior can be seen in figure 3 where $\operatorname{Ln} I$ is plotted as a function of $\operatorname{Ln}\left(V-V_{g}\right)$ : the slope of the curve changes from 2 at moderate voltages to 1 at high voltages.

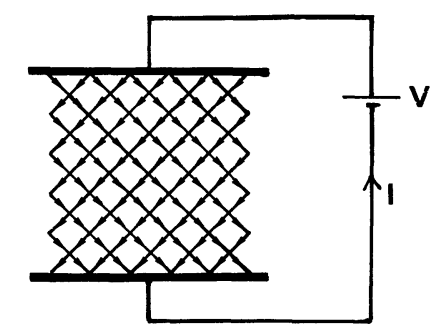

Fig.1.- On each link of the square lattice shown are distributed at random a Zener diode in the forward direction and a $1 \mathrm{k} \Omega$ resistance mounted in series. 


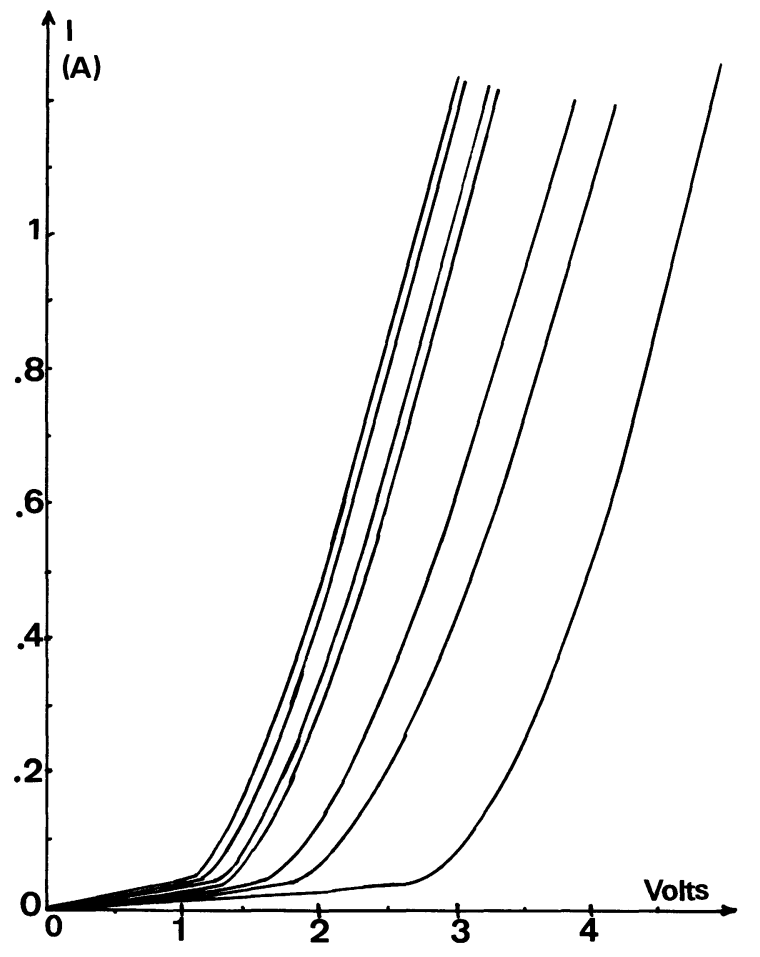

Fig.2.- Different $i(v)$ characteristic of the element of the links.

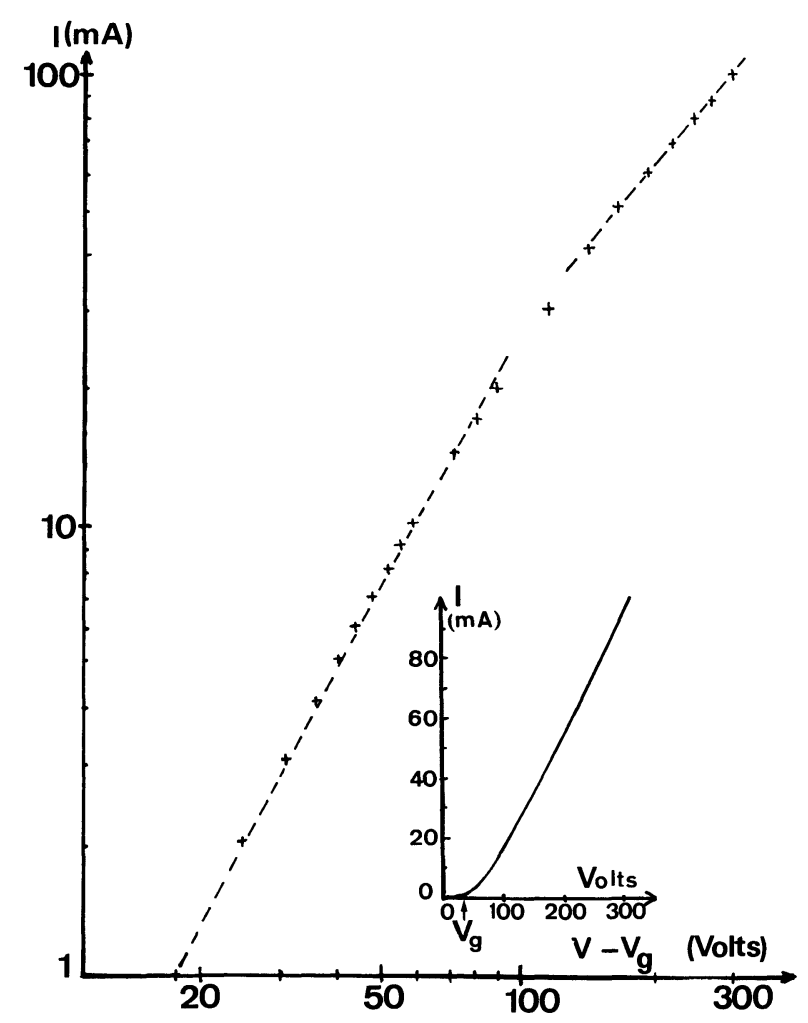

Fig.3.- a) Insert : $I(\eta)$ characteristic of a network of size $10 \times$ 24 éléments. b) $I$ versus $\left(V-V_{\mathrm{g}}\right)$ in logarithmic scale.
This result was checked to not depend on the size of the network.

At high voltage, each diode is in the linear regime. Therefore, the total network regime is also linear. At intermediate voltage, some diodes are highly resistive $\left(v<v_{\mathrm{g}}\right)$ while others are conducting $\left(v>v_{\mathrm{g}}\right)$. In this "percolating" regime, the behaviour of the total network is non linear. The quadratic dependence of the current $I$ on the voltage $V$ is in good agreement with the numerical simulation of Roux and Herrmann [6] and comes from the increase of the number of active diodes with $V$. The exponent 2 is also consistent with a mean field solution of the problem. At sufficiently low voltage, each diode is in the highly resistive regime $\left(v<v_{\mathrm{g}}\right)$, such that almost no current flows through the network. An analogy between the behaviour above $V_{\mathrm{g}}$ and the critical one around percolation threshold has been proposed recently $[7,8]$. This result cannot be compared directly with the simulations of Roux and Herrmann, as these authors have considered a distribution of threshold voltages $v_{\mathrm{g}}$ down to zero while in our experiments, the minimum value of $v_{\mathbf{g}}$ is finite. Another major difference lies in the fact that the Zener diodes used are not symmetric with respect to the direction of the current flowing through them. Therefore, the problem we studied here is somewhat analogous to a "directed" case (the current cannot flow backward). However, the directed and non-directed cases should not be very different as suggested in some recent numerical results [8]. This comes from the fact that a certain amount of "directedness" is naturally provided by the propagation of the current itself : it can hardly flow "backward" (with respect to the mean voltage drop direction) for the low values of "disorder" (width of the distribution) of thresholds $v_{\mathrm{g}}$ we chose. For larger distributions $P\left(v_{\mathrm{g}}\right)$ of threshold (having for instance a singularity at $v_{\mathrm{g}}=0$ i.e. $P\left(v_{\mathrm{g}}\right) \propto v_{\mathrm{g}}{ }^{-x}$ with an exponent $0<x<1$ ) then, the mean path followed by the current could be much more tortuous than in our case.

In conclusion, our experimental results on a two dimensional random diode network, confirm the numerical simulations of Roux and Herrmann [6] . It is worth pointing out that the above results can have more general applications. The non linear network we have considered, could roughly simulate the outflow of a Bingham fluid though a porous medium where the flow and the pressure applied to the fluid correspond respectively to the current and the voltage. Similarly, the diode network current-voltage characteristic could be related to the stress-strain relation of a piling of cylinders with fluctuating radii $[9,10]$. 


\section{Acknowledgments \\ We would like to thank Dr C. Vanneste and Dr D. Sornette for stimulating discussions.}

\section{References}

[1] de Arcangelis, L., Redner, S., and HerRMANN, H.J., J. Physique Lett. 46 (1985) L 585.

[2] Takayasu, H., Phys. Rev. Lett. 54 (1985) 1099.

[3] Gilabert, A., Vanneste, C. Sornette, D., et GUYON, E., J. Physique 48 (1987) 763.

[4] REDner, S., Ecole de Physique Théorique, Les Houches (1986).

[5] Coniglio, A., On Growth and Form, Edited by H.E. Stanley and N. Ostrowsky (Martinus Nijhoff Publishers) (1986) p.101.
[6] Roux, S., and Herrmann, H.J., To be published.

[7] Roux, S., Herrmann, H.J., Hansen, A., and GuYon, E., C.R.Heb. Séan. Acad. Sci. To be published.

[8] Roux, S., Hansen, A., and Guyon, E., To be published.

[9] Travers, T., Bideau, D., Gervois, A., TroADEC, J.P. and MESSAger, J.C., J. Phys. A Lett. 19 (1986) L 1033.

[10] HerrmanN, H.J., STAUfFer, D. and RouX, S., J. Physique 48 (1987) 346. 